\title{
Singularities in Dromo formulation. Analysis of deep flybys
}

\author{
Javier Roa $^{\mathrm{a}, *}$, Manuel Sanjurjo-Rivo ${ }^{\mathrm{b}}$, Jesús Peláez ${ }^{\mathrm{a}}$ \\ ${ }^{a}$ Space Dynamics Group, Technical University of Madrid, Pza. Cardenal Cisneros 3, E-28040 Madrid, Spain \\ ${ }^{\mathrm{b}}$ Bioengineering and Aerospace Engineering Dept., Carlos III University, Avda. de la Universidad 30, E-28911 Leganés, Madrid, Spain
}

\begin{abstract}
The singularities in Dromo are characterized in this paper, both from an analytical and a numerical perspective. When the angular momentum vanishes, Dromo may encounter a singularity in the evolution equations. The cancellation of the angular momentum occurs in very specific situations and may be caused by the action of strong perturbations. The gravitational attraction of a perturbing planet may lead to rapid changes in the angular momentum of the particle. In practice, this situation may be encountered during deep planetocentric flybys. The performance of Dromo is evaluated in different scenarios. First, Dromo is validated for integrating the orbit of Near Earth Asteroids. Resulting errors are of the order of the diameter of the asteroid. Second, a set of theoretical flybys are designed for analyzing the performance of the formulation in the vicinity of the singularity. New sets of Dromo variables are proposed in order to minimize the dependency of Dromo on the angular momentum. A slower time scale is introduced, leading to a more stable description of the flyby phase. Improvements in the overall performance of the algorithm are observed when integrating orbits close to the singularity. (C) 2015 COSPAR. Published by Elsevier Ltd. All rights reserved.
\end{abstract}

\section{Introduction}

Monitoring the trajectories of Near Earth Asteroids (NEAs) is critical for evaluating their collision probability. Milani et al. (2000) propose a method for predicting future asteroid close approaches, considering resonant returns. A detailed analysis of such resonances may be found in the work by Kozai (1985). Valsecchi et al. (2003) develop an analytical theory for deriving the initial conditions that yield a particular geometry of the close approach, defined by a set of coordinates on the $b$-plane. Close approaches to the major planets may modify substantially the heliocentric orbit of the asteroid. An accurate description of the planetary flybys is required for predicting future

\footnotetext{
* Corresponding author at: Jet Propulsion Laboratory, California Institute of Technology, 4800 Oak Grove Drive, Pasadena, CA 91109, USA.

E-mail address: javier.roa@upm.es (J. Roa).
}

encounters, and for reducing the uncertainties in the observations. An overview of the techniques for merging observations and computations can be found in the work by Bowell et al. (2002).

The first approach to solving interplanetary trajectories is the patched-conics (or linked-conics) solution. In this approach the orbit is assumed to be Keplerian, neglecting external perturbations. Minovitch (1961) formally stated the hyperbolic motion of a particle about a certain planet and his work became the basis of future works on the subject. The patched-conics technique simplifies the preliminary analyses and allows to define the corresponding encounters. Flandro (1966) studied the maximum energy increment and maximum deflection angle for a spacecraft flying about the major planets. Kemble (2006) provides an exhaustive description of different design techniques in his book. Analytical techniques provide valuable information about the properties of the problem. Ross and Scheeres (2007) defined the Keplerian map, that transforms 
a set of Keplerian elements before the flyby to the set of Keplerian elements after the flyby. The perturbations on an asteroid or spacecraft navigating the Solar System are considered when the trajectory of the particle needs to be propagated accurately. Prado (2007) compares the error in the energy increment of a particle during jovicentric flybys when computed with the patched-conics solution and the full numerical integration. Campagnola et al. (2012) advanced on the concept of the Keplerian map and built the Flyby Map via numerical integration of the three-body problem.

The scientific importance of interplanetary trajectories and close encounters matches the complexity of their computation. The intrinsic Lyapunov local instability of Keplerian motion makes errors in orbit determination to propagate rapidly. Bond (1982) analyzed exhaustively the error growth rate of Cowell's and Encke's method. Such behavior is particularly critical when propagating flyby trajectories, due to the rapid changes in the dynamics of the particle. The unstable eigenvalue of the linearized system grows as the perigee height decreases, so numerical integration may suffer during deep flybys. There have been reported anomalies in the asymptotic velocities of a number of spacecraft after a flyby. Anderson et al. (2008) found an anomalous energy change in the geocentric flybys of Galileo I, NEAR, and Rosetta, derived from an unexpected Doppler shift. They attribute this energy change to an unexpected perturbation due to the rotation of the Earth, that may be larger than the predicted frame-dragging effect. Rievers and Lämmerzahl (2011) derived a detailed thermal model in an attempt to explain the anomalous acceleration detected on Pioneer 10 and 11 spacecraft. Acedo (2014) provides a wide overview of detected anomalies during flybys, and the explanatory theories that have been proposed. He analyzes the effect of the gravitomagnetic field on the motion of the particle and its impact on the dynamics of the flyby. More complex theories have been formulated: Adler (2009) considered the possible relation of dark-matter surrounding the Earth with the anomalous velocities detected during geocentric flybys. To this level of accuracy high fidelity numerical propagation models are required when fitting observational data.

The evolution of the available ephemeris is a clear proof of how new missions require improved precisions. The JPL has just released the series of planetary ephemeris DE430 (Folkner et al., 2014): recent missions to Mercury, Mars and Saturn require a more precise estimation of the dynamical state of these planets, which is the major advance of the DE430 ephemeris with respect to the previous DE405 and DE421. New Horizons mission is expected to reach Pluto by 2015. To fulfill the scientific goals of the mission the precision in determining Pluto's orbit is crucial. For this purpose, the planetary ephemeris DE432 includes an update of the estimated orbit of Pluto (Folkner, 2014). But in order to improve the overall accuracy of the numerical propagation algorithms not only more detailed force models are required; the performance of the formulation and the integration scheme should also be considered.

Sophisticated propagation methods are typically related to regularization. Different approaches to regularizing the equations of orbital motion are found in the literature. Levi-Civita (1920) introduced a transformation in the complex plane to formulate the planar problem. The LeviCivita variables were extended to the three dimensional space by Kustaanheimo and Stiefel (1965). Burdet (1968), based on previous work by Sperling (1961), embedded the Laplace vector and the energy in the equations of motion arriving to a fully regularized formulation. These formulations all share a change of variable: the physical time is replaced by a fictitious time via the Sundman transformation (Bond and Allman, 1996, Chap. 9). The resulting governing equations reduce to a harmonic oscillator in the unperturbed case, including a forcing term in the Sperling-Burdet formulation. These equations are linear and decoupled, a major advantage when compared to Cowell's formulation. Laplace et al. (1843) had already explored the linear form of the equations of orbital motion. Laplace's work and its connection with the KustaanheimoStiefel and Sperling-Burdet regularizations are analyzed in detail by Deprit et al. (1994).

Deprit (1976) recovered the concept of Hansen ideal reference frames (Hansen, 1853) to refer the perturbed two-body problem to an ideal reference frame. Palacios and Calvo (1996) advanced on Deprit's work and proposed a regularization of the equations of motion based on ideal frames. These concepts and the introduction of the fictitious time motivated Peláez et al. (2007) to derive a special perturbation method, called Dromo. Dromo first introduces a Hansen ideal reference frame that describes the motion of the orbital plane. The evolution of the orbital plane is defined by a quaternion. Second, the motion of the particle on the orbital plane is described, accounting for the evolution of the osculating eccentricity vector. Dromo formulation has been tested in different scenarios with promising results (Baù and Bombardelli, 2014). There is a renewed interest in extending Dromo to the propagation of flyby trajectories. A singularity in the equations of Dromo has been detected in very specific flyby scenarios, and requires a dedicated analysis. Roa and Peláez (2015) propose a reformulation of Dromo specifically conceived for hyperbolic orbits, and Baù et al. (2014) focus on the elliptic problem. These particular formulations enhance the performance of the method, but they are restricted to the hyperbolic and elliptic cases, respectively. There is still a lack of specific studies on the potential singularities.

This paper pursues two main objectives. First, the singularities in Dromo formulation are explored, in order to fully characterize their behavior. Second, alternative formulations to mitigate the effect of the singularities are proposed. Following the present Introduction, Section 2 provides a detailed description of Dromo equations. Next section addresses the singularities of Dromo. Once the singularities have been introduced, two alternative 
formulations are proposed in Section 4. Finally, the numerical performance of Dromo is studied through a set of numerical examples. The orbits of three NEAs are integrated and compared against the solution provided by $\mathrm{JPL} /$ Horizons, in order to validate the application of Dromo in such scenarios.

\section{Dromo formulation}

Peláez et al. (2007) developed a special perturbation method which they called Dromo. They regularize the equations of motion by means of a Sundman transformation. The dynamics of the particle are decomposed in the motion in the orbital plane and the motion of the orbital plane itself. This concept has also been studied by Deprit (1976) and comes from the definition of the Hansen ideal reference frames (Hansen, 1853, p. 66). The orbital plane is defined using a quaternion instead of the classical Euler angles. The formulation is valid for all kinds of non-degenerate orbits; circular, elliptic, parabolic, and hyperbolic. The formulation discussed in this work corresponds to a more recent version of Dromo posed by Urrutxua et al. (2013).

Dromo was conceived as a high-performance propagator to integrate weakly perturbed trajectories. Urrutxua and Peláez (2012) and Baù and Bombardelli (2014) have conducted a wide variety of experiments to evaluate the performance of the algorithm. The set of Dromo elements are particularly interesting due to their geometrical and kinematical meaning. Based on them, Roa and Peláez (2014) arrived to a new concept of transformation for solving the elliptic rendezvous problem. Bombardelli et al. (2011) and Gonzalo and Bombardelli (2014) proposed asymptotic solutions to the tangential and radial constant thrust problem based on the Dromo elements. Attention has been paid to the propagation of flyby trajectories with Dromo in different scenarios (Roa and Peláez, 2015).

\subsection{Equations of motion}

Let $I$ denote an inertial reference frame, defined by the basis $I=\left\{C: \mathbf{i}_{I}, \mathbf{j}_{I}, \mathbf{k}_{I}\right\}$, and let $\mathbf{r} \in \mathbb{R}^{3}$ be the radius-vector of a particle orbiting the attractive center $C$. The time evolution of the radius-vector is given by

$\frac{\mathrm{d}^{2} \hat{\mathbf{r}}}{\mathrm{d} t^{2}}+\frac{\hat{\mu}}{\|\hat{\mathbf{r}}\|^{3}} \hat{\mathbf{r}}=\hat{\mathbf{a}}_{\mathrm{p}}$,

where $(\hat{\bullet})$ denotes dimensional variables, $\hat{\mu}$ is the gravitational parameter, and $\hat{\mathbf{a}}_{\mathrm{p}}$ accounts for external perturbing accelerations. Under a normalization such that $\mu=1$, the mean motion is $n=1$, and the semimajor axis is $a=1$, the governing equation reduces to

$\frac{\mathrm{d}^{2} \mathbf{r}}{\mathrm{d} \tau^{2}}+\frac{\mathbf{r}}{r^{3}}=\mathbf{a}_{\mathrm{p}}, \quad r=\|\mathbf{r}\|$, with $\tau$ the normalized time.

Let $\mathcal{L}$ be an orbital frame centered at $\mathcal{C}$, with $\mathcal{L}=\left\{\mathcal{C} ; \mathbf{i}_{\mathcal{L}}, \mathbf{j}_{\mathcal{L}}, \mathbf{k}_{\mathcal{L}}\right\}$. Such basis is defined by the expressions:

$\mathbf{i}_{\mathcal{L}}=\frac{\mathbf{r}}{r}, \quad \mathbf{k}_{\mathcal{L}}=\frac{\mathbf{h}}{\|\mathbf{h}\|}=\frac{\mathbf{h}}{h}, \quad \mathbf{j}_{\mathcal{L}}=\mathbf{k}_{\mathcal{L}} \times \mathbf{i}_{\mathcal{L}}$,

where $\mathbf{h} \in \mathbb{R}^{3}$ is the angular momentum vector of the particle.

Consider a particular perifocal reference, namely the departure perifocal frame $\mathcal{P}=\left\{C: \mathbf{i}_{\mathcal{P}}, \mathbf{j}_{\mathcal{P}}, \mathbf{k}_{\mathcal{P}}\right\}$, defined by

$\mathbf{i}_{\mathcal{\rho}}=\frac{\mathbf{e}_{0}}{e_{0}}, \quad \mathbf{k}_{\mathcal{P}}=\frac{\mathbf{h}_{0}}{h_{0}}, \quad \mathbf{j}_{\mathcal{\rho}}=\mathbf{k}_{\mathcal{\rho}} \times \mathbf{i}_{\mathcal{\rho}}$,

with $\mathbf{e}_{0}$ and $\mathbf{h}_{0}$ the eccentricity and angular momentum vectors at departure, respectively. Frame $\mathcal{P}$ is attached to the orbital plane and describes its dynamics. The definition of this reference frame is not arbitrary; consider the following theorem, proposed by Hansen (1853) and further discussed by Jochim (2012):

Theorem 1. A reference frame is said to be Hansen ideal if and only if the absolute velocity vector of the particle always coincide with the relative velocity vector.

Corollary. The departure perifocal frame $\mathcal{P}$ defines a Hansen ideal reference frame.

Proof. The absolute velocity vector of the particle relates to the relative velocity vector by means of the angular velocity of frame $\mathcal{P}$ w.r.t. the inertial reference $\mathcal{I}, \omega_{\mathcal{P}_{I}}$,

$\left.\frac{\mathrm{d} \mathbf{r}}{\mathrm{d} \tau}\right|_{I}=\left.\frac{\mathrm{d} \mathbf{r}}{\mathrm{d} \tau}\right|_{\mathcal{P}}+\omega_{\mathcal{P}_{I}} \times \mathbf{r}$.

Thus, proof of Theorem 1 relies on proving that $\omega_{\mathscr{P}_{I}} \mid \mathbf{r}$.

The time derivative of versor $\mathbf{i}_{\mathcal{L}}$ abides by

$\left.\frac{\mathrm{d} \mathbf{i}_{\mathcal{L}}}{\mathrm{d} \tau}\right|_{I}=\omega_{\mathcal{L} I} \times \mathbf{i}_{\mathcal{L}}$.

From the definition of the basis $\left\{\mathbf{i}_{\mathcal{L}}, \mathbf{i}_{\mathcal{L}}, \mathbf{k}_{\mathcal{L}}\right\}$ given in Eq. (1) the previous time derivative admits an alternative expression:

$\left.\frac{\mathrm{d} \mathbf{i}_{\mathcal{L}}}{\mathrm{d} \tau}\right|_{I}=\frac{\mathrm{d}}{\mathrm{d} \tau}\left(\frac{\mathbf{r}}{r}\right)=\frac{1}{r}\left(\mathbf{v}-\frac{\mathrm{d} r}{\mathrm{~d} \tau} \mathbf{i}_{\mathcal{L}}\right)$,

where $\mathbf{v}$ is the velocity vector of the particle. This expression yields the equality

$\frac{1}{r}\left(\mathbf{v}-\frac{\mathrm{d} r}{\mathrm{~d} \tau} \mathbf{i}_{\mathcal{L}}\right)=\boldsymbol{\omega}_{\mathcal{L} \mathcal{L}} \times \mathbf{i}_{\mathcal{L}}$.

In a similar fashion, the time derivative of versor $\mathbf{k}_{\mathcal{L}}$ results in

$\left.\frac{\mathrm{d} \mathbf{k}_{\mathcal{L}}}{\mathrm{d} \tau}\right|_{I}=\omega_{\mathcal{L I}} \times \mathbf{k}_{\mathcal{L}}=\frac{\mathrm{d}}{\mathrm{d} \tau}\left(\frac{\mathbf{h}}{h}\right)=\frac{1}{h}\left(\frac{\mathrm{d} \mathbf{h}}{\mathrm{d} \tau}-\frac{\mathrm{d} h}{\mathrm{~d} \tau} \mathbf{k}_{\mathcal{L}}\right)$. 
Assume that the angular velocity $\omega_{\mathcal{L} I}$ is written in terms of its components in the orbital frame $\mathcal{L}$ as $\omega_{\mathcal{L} I}=\omega_{x} \mathbf{i}_{\mathcal{L}}+$ $\omega_{y} \mathbf{j}_{\mathcal{L}}+\omega_{z} \mathbf{k}_{\mathcal{L}}$. In this form, Eqs. (3) and (4) become

$\frac{1}{r}\left(\mathbf{v}-\frac{\mathrm{d} r}{\mathrm{~d} \tau} \mathbf{i}_{\mathcal{L}}\right)=\omega_{z} \mathbf{j}_{\mathcal{L}}-\omega_{y} \mathbf{k}_{\mathcal{L}}$

$\frac{1}{h}\left(\frac{\mathrm{dh}}{\mathrm{d} \tau}-\frac{\mathrm{d} h}{\mathrm{~d} \tau} \mathbf{k}_{\mathcal{L}}\right)=\omega_{y} \mathbf{i}_{\mathcal{L}}-\omega_{x} \mathbf{i}_{\mathcal{L}}$.

These equations are projected onto the orbital frame $\mathcal{L}$ to provide:

$\omega_{x}=-\frac{1}{h}\left(\frac{\mathrm{d} \mathbf{h}}{\mathrm{d} \tau} \cdot \mathbf{j}_{\mathcal{L}}\right)=-\frac{1}{h}\left(\mathbf{r} \times \mathbf{a}_{\mathrm{p}}\right) \cdot \mathbf{j}_{\mathcal{L}}=\frac{r}{h}\left(\mathbf{a}_{\mathrm{p}} \cdot \mathbf{k}_{\mathcal{L}}\right)$,

$\omega_{y}=0$,

$\omega_{z}=\frac{1}{r}\left(\mathbf{v} \cdot \mathbf{j}_{\mathcal{L}}\right)=-\frac{1}{r} \mathbf{v} \cdot\left(\mathbf{i}_{\mathcal{L}} \times \mathbf{k}_{\mathcal{L}}\right)=\frac{1}{r^{2}}(\mathbf{k} \cdot \mathbf{h})=\frac{h}{r^{2}}$.

The angular velocity of the orbital reference $\mathcal{L}$ is then written

$\boldsymbol{\omega}_{\mathcal{L} T}=\frac{r}{h}\left(\mathbf{a}_{\mathrm{p}} \cdot \mathbf{k}_{\mathcal{L}}\right) \mathbf{i}_{\mathcal{L}}+\frac{h}{r^{2}} \mathbf{k}_{\mathcal{L}}$.

Note that the second term corresponds to the in-plane motion of the particle, $\omega_{\mathcal{L}^{\rho}}=h / r^{2} \mathbf{k}_{\mathcal{L}}$. That is,

$\omega_{\mathcal{L}}=\frac{h}{r^{2}} \mathbf{k}_{\mathcal{L}}, \quad$ and $\quad \omega_{\mathcal{P}_{I}}=\frac{r}{h}\left(\mathbf{a}_{\mathrm{p}} \cdot \mathbf{k}_{\mathcal{L}}\right) \mathbf{i}_{\mathcal{L}}$.

Provided that $\mathbf{r}=r \mathbf{i}_{\mathcal{L}}$, it is $\boldsymbol{\omega}_{\mathcal{P}_{I}} \times \mathbf{r}=0$ and Eq. (2) reduces to

$\left.\frac{\mathrm{d} \mathbf{r}}{\mathrm{d} \tau}\right|_{I}=\left.\frac{\mathrm{d} \mathbf{r}}{\mathrm{d} \tau}\right|_{\mathcal{P}}$.

The absolute velocity vector is equal to the relative velocity vector, Theorem 1 holds and $\mathcal{P}$ is a Hansen ideal reference frame.

The independent variable in Dromo is the ideal anomaly, $\sigma$, defined as

$\sigma=\beta+\theta$,

with $\beta$ the angle between the osculating eccentricity vector and $\mathbf{e}_{0}$, and $\theta$ the true anomaly. The ideal anomaly behaves as a fictitious time considering the transformation

$\frac{\mathrm{d} \tau}{\mathrm{d} \sigma}=\frac{r^{2}}{h}=\zeta_{3} r^{2}$,

with $\zeta_{3}=1 / h$.

Figure 1 shows the geometry of the different reference frames that have been introduced. The relation between the ideal anomaly and the true anomaly can be observed in this figure.

\subsection{The eccentricity vector}

The definition of the eccentricity vector, $\mathbf{e}$, is

$\mathbf{e}=\frac{\mathbf{v} \times \mathbf{h}}{\mu}-\frac{\mathbf{r}}{r}$.

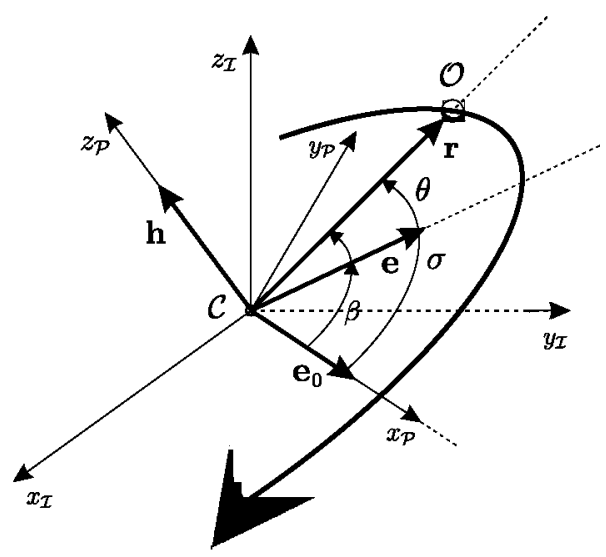

Fig. 1. Geometrical definition of the problem.

Provided that the velocity is given by

$\mathbf{v}=\frac{\mathrm{d} \mathbf{r}}{\mathrm{d} \tau}=\frac{\mathrm{d} r}{\mathrm{~d} \tau} \mathbf{i}_{\mathcal{L}}+\frac{h}{r} \mathbf{j}_{\mathcal{L}}$,

Eq. (7) translates into:

$\mathbf{e}=\left(\frac{h^{2}}{r}-1\right) \mathbf{i}_{\mathcal{L}}-h \frac{\mathrm{d} r}{\mathrm{~d} \tau} \mathbf{j}_{\mathcal{L}}$.

This expression is written in the departure perifocal frame $\mathcal{P}$ to provide:

$$
\begin{aligned}
\mathbf{e}= & {\left[\left(\frac{h^{2}}{r}-1\right) \cos \sigma+h \frac{\mathrm{d} r}{\mathrm{~d} \tau} \sin \sigma\right] \mathbf{i}_{\mathcal{P}} } \\
& +\left[\left(\frac{h^{2}}{r}-1\right) \sin \sigma-h \frac{\mathrm{d} r}{\mathrm{~d} \tau} \cos \sigma\right] \mathbf{j}_{\mathcal{P}}=\zeta_{1} \mathbf{i}_{\rho}+\zeta_{2} \mathbf{j}_{\mathcal{\rho}} .
\end{aligned}
$$

That is, $\left(\zeta_{1}, \zeta_{2}\right) \in \mathbb{R}^{2}$ define the components of the eccentricity vector in the departure perifocal frame $\mathcal{P}$. Recalling how the angle $\beta$ describes the evolution of the osculating eccentricity vector, $\zeta_{1}$ and $\zeta_{2}$ admit two alternative expressions:

$$
\begin{aligned}
& \zeta_{1}=\left(\frac{h^{2}}{r}-1\right) \cos \sigma+h \frac{\mathrm{d} r}{\mathrm{~d} \tau} \sin \sigma \equiv e \cos \beta, \\
& \zeta_{2}=\left(\frac{h^{2}}{r}-1\right) \sin \sigma-h \frac{\mathrm{d} r}{\mathrm{~d} \tau} \cos \sigma \equiv e \sin \beta .
\end{aligned}
$$

Recall that

$\zeta_{3}=\frac{1}{h}$.

The triple $\left(\zeta_{1}, \zeta_{2}, \zeta_{3}\right) \in \mathbb{R}^{3}$ defines the first three Dromo elements. In the unperturbed case it is $\beta=0$, and therefore $\zeta_{1}=e$ and $\zeta_{2}=0$ for all $\tau$.

\subsection{The orbital plane}

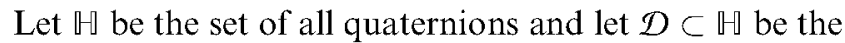
subset $\mathcal{D}=\{\mathbf{q} \in \mathbb{H} \mid \Re(\mathbf{q})=0\}$ of all pure quaternions. $A$ quaternion $\mathrm{q} \in \mathbb{H}$ decomposes in the scalar part, $q_{0}=\mathfrak{R}(\mathbf{q})$, and the vector part, $\mathbf{q}=\left(q_{1}, q_{2}, q_{3}\right)$, where 
$\left(q_{0}, q_{1}, q_{2}, q_{3}\right) \in \mathbb{R}^{4}$ are the components of quaternion $q$. Let

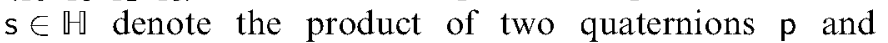
$\mathrm{q}, \mathrm{s}=\mathrm{pq}$. The scalar and vector parts of $\mathrm{s}$ are, respectively, $s_{0}=p_{0} q_{0}-\mathbf{p} \cdot \mathbf{q}, \quad$ and $\quad \mathbf{s}=p_{0} \mathbf{q}+q_{0} \mathbf{p}+\mathbf{p} \times \mathbf{q}$.

Remark. Multiplication is not commutative in $\mathbb{U}$.

Consider that $\mathbf{r}_{I} \in \mathcal{D}$ and $\mathrm{r}_{\mathcal{P}} \in \mathcal{D}$ are two pure quaternions associated with the components of vector $\mathbf{r}$ in the inertial $I$ and in the departure perifocal $\mathcal{P}$ reference frames, respectively. Both quaternions relate through the rotation action

$\mathrm{r}_{\bar{I}}=\mathrm{n} \mathrm{r}_{\mathcal{P}} \tilde{\mathrm{n}}, \quad \mathrm{n} \in \mathbb{H}$.

Quaternion $\tilde{\mathbf{n}}$ is the conjugate quaternion of $\mathbf{n}$. Provided that rotations preserve the norm, it is $\left\|\mathbf{r}_{T}\right\|=\left\|\mathbf{r}_{\varphi}\right\|$ and consequently $\left|r_{I}\right|=\left|r_{\mathcal{P}}\right|$.

Proposition 1. Eq. (8) preserves the modulus of quaternion $\mathbf{r}_{\mathcal{P}}$ if and only if quaternion $\mathrm{n}$ is unitary.

Proof. The modulus of $r_{I}$ is obtained from Eq. (8), considering the conjugate quaternion $\tilde{\mathbf{r}}_{I}=n \tilde{\mathbf{r}}_{p} \tilde{\mathrm{n}}$ :

$$
\begin{aligned}
\left|\mathbf{r}_{I}\right|^{2} & =\mathbf{r}_{I} \tilde{r}_{I}=\left(\mathbf{n} r_{\mathcal{P}} \tilde{\mathbf{n}}\right)\left(\mathbf{n} \tilde{\mathbf{r}}_{\mathcal{P}} \tilde{\mathbf{n}}\right)=\left(\mathbf{n} \mathbf{r}_{\mathcal{P}}\right)\left(\tilde{\mathbf{r}}_{\mathcal{P}} \tilde{\mathbf{n}}\right)|\mathbf{n}|^{2} \\
& =(\mathbf{n} \tilde{\mathbf{n}})|\mathbf{n}|^{2}\left|\mathbf{r}_{\mathcal{P}}\right|^{2}=|\mathbf{n}|^{4}\left|\mathbf{r}_{\mathcal{P}}\right|^{2},
\end{aligned}
$$

and therefore

$\left|\mathbf{r}_{I}\right|=\left|\mathbf{r}_{\mathcal{P}}\right| \Longleftrightarrow|\mathbf{n}|=1$.

Quaternion $\mathbf{n}=\eta_{4}+\mathrm{i} \eta_{1}+\mathrm{j} \eta_{2}+\mathrm{k} \eta_{3}$ relates to the Euler angles by means of successive applications of Euler's Theorem:

$\eta_{1}=\sin \frac{i}{2} \cos \frac{\Omega-\tilde{\omega}}{2}, \quad \eta_{2}=\sin \frac{i}{2} \sin \frac{\Omega-\tilde{\omega}}{2}$,

$\eta_{3}=\cos \frac{i}{2} \sin \frac{\Omega+\tilde{\omega}}{2}, \quad \eta_{4}=\cos \frac{i}{2} \cos \frac{\Omega+\tilde{\omega}}{2}$,

with $i$ the osculating inclination, $\Omega$ the osculating right ascension of the ascending node, and $\tilde{\omega}$ the departure argument of the pericenter. The Euler parameters $\left(\eta_{1}, \eta_{2}, \eta_{3}, \eta_{4}\right) \in \mathbb{R}^{4}$ are the last four Dromo elements.

Conclusion: The set of Dromo elements is $\mathbf{D}=\left(\zeta_{1}, \zeta_{2}, \zeta_{3}, \eta_{1}, \eta_{2}, \eta_{3}, \eta_{4}\right)^{\top}$, with $\mathbf{D} \in \mathbb{R}^{7}$.

\subsection{Evolution equations}

The distance from the particle $O$ to the attractive center $C$ is given by

$r=\frac{h^{2}}{1+e \cos \theta}=\frac{1}{\zeta_{3}^{2}(1+e \cos \theta)}=\frac{1}{\zeta_{3}^{2} s}$

with

$s=1+e \cos (\sigma-\beta)=1+e \cos \beta \cos \sigma+e \sin \beta \sin \sigma$

$=1+\zeta_{1} \cos \sigma+\zeta_{2} \sin \sigma$.
The physical time is defined by means of

$\frac{\mathrm{d} \tau}{\mathrm{d} \sigma}=\frac{1}{\zeta_{3}^{3} s^{2}}$.

Recall that the time $\tau$ is a dependent variable in Dromo.

The hodograph equation is obtained from the cross product of $\mathbf{k}_{\mathcal{L}}$ and the eccentricity vector, given by Eq. (7). It reads

$\mathbf{v}=\zeta_{3}\left(\mathbf{j}_{\mathcal{L}}+\mathbf{k}_{\mathcal{L}} \times \mathbf{e}\right)$.

Considering that $\mathbf{e}=\zeta_{1} \mathbf{i}_{\rho}+\zeta_{2} \mathbf{j}_{\mathcal{p}}$, it yields

$\zeta_{1} \mathbf{j}_{\rho}-\zeta_{2} \mathbf{i}_{\rho}=-\mathbf{j}_{\mathcal{L}}+\frac{\mathbf{v}}{\zeta_{3}}$.

This equation is differentiated w.r.t. the ideal anomaly $\sigma$ and projected along $\mathbf{i}_{\rho}$ and $\mathbf{j}_{\rho}$ to provide

$\frac{\mathrm{d} \zeta_{1}}{\mathrm{~d} \sigma}=+s \sin \sigma\left(\mathbf{a}_{\mathrm{p}}^{*} \cdot \mathbf{i}_{\mathcal{L}}\right)+\left[\zeta_{1}+(1+s) \cos \sigma\right]\left(\mathbf{a}_{\mathrm{p}}^{*} \cdot \mathbf{j}_{\mathcal{L}}\right)$

$\frac{\mathrm{d} \zeta_{2}}{\mathrm{~d} \sigma}=-s \cos \sigma\left(\mathbf{a}_{\mathrm{p}}^{*} \cdot \mathbf{i}_{\mathcal{L}}\right)+\left[\zeta_{2}+(1+s) \sin \sigma\right]\left(\mathbf{a}_{\mathrm{p}}^{*} \cdot \mathbf{j}_{\mathcal{L}}\right)$

where the term $\mathbf{a}_{\mathrm{p}}^{*}$ stands for $\mathbf{a}_{\mathrm{p}}^{*}=\mathbf{a}_{\mathrm{p}} /\left(\zeta_{3}^{4} s^{3}\right)$.

The angular momentum vector changes according to the expression

$\frac{\mathrm{d} \mathbf{h}}{\mathrm{d} \tau}=\mathbf{r} \times \mathbf{a}_{\mathrm{p}}=r\left(\mathbf{i}_{\mathcal{L}} \times \mathbf{a}_{\mathrm{p}}\right)$

where it is $\mathbf{h}=h \mathbf{k}_{\mathcal{L}}$. Considering that

$\begin{aligned} \frac{\mathrm{d} \mathbf{h}}{\mathrm{d} \tau} & =\frac{\mathrm{d} h}{\mathrm{~d} \tau} \mathbf{k}_{\mathcal{L}}+h \frac{\mathrm{d} \mathbf{k}_{\mathcal{L}}}{\mathrm{d} \tau}=\frac{\mathrm{d} h}{\mathrm{~d} \tau} \mathbf{k}_{\mathcal{L}}+h\left(\omega_{\mathcal{L} \mathcal{T}} \times \mathbf{k}_{\mathcal{L}}\right) \\ & =\frac{\mathrm{d} h}{\mathrm{~d} \tau} \mathbf{k}_{\mathcal{L}}-r\left(\mathbf{a}_{\mathrm{p}} \cdot \mathbf{k}_{\mathcal{L}}\right) \mathbf{j}_{\mathcal{L}},\end{aligned}$

the evolution equation for $\mathbf{h}$ is projected along $\mathbf{k}_{\mathcal{L}}$ to provide

$\frac{\mathrm{d} h}{\mathrm{~d} \tau}=r\left(\mathbf{a}_{\mathrm{p}} \cdot \mathbf{j}_{\mathcal{L}}\right) \Rightarrow \frac{\mathrm{d} h}{\mathrm{~d} \sigma}=\frac{r^{3}}{h}\left(\mathbf{a}_{\mathrm{p}} \cdot \mathbf{j}_{\mathcal{L}}\right)$.

The derivative of $\zeta_{3}$ results in

$\frac{\mathrm{d} \zeta_{3}}{\mathrm{~d} \sigma}=-\zeta_{3}\left(\mathbf{a}_{\mathrm{p}}^{*} \cdot \mathbf{j}_{\mathcal{L}}\right)$.

That is, the angular momentum of the particle is only affected by the projection of the perturbing acceleration in the along-track direction.

Let $w_{\mathcal{P}_{I}} \in \mathcal{D}$ be the pure quaternion defined by the projections of vector $\omega_{\mathscr{P}_{I}} / 2$ in frame $\mathcal{P}$. This quaternion relates to quaternion $n$ by means of

$2 w_{\mathcal{P}_{I}}=2 \tilde{\mathrm{n}} \frac{\mathrm{d} \mathrm{n}}{\mathrm{d} \tau}$.

This equation is inverted to provide the time evolution of quaternion $\mathrm{n}$,

$\frac{\mathrm{dn}}{\mathrm{d} \tau}=\frac{1}{2} \mathrm{n}\left(2 \mathrm{w}_{\rho_{I}}\right)$.

Expanding the quaternion product and taking into account the definition of $\omega_{\mathcal{P}_{I}}$ given in Eq. (5) provides the evolution 
equations for the components of $\mathrm{n}$. The full system of eight ordinary differential equations becomes

$$
\begin{aligned}
& \frac{\mathrm{d} \tau}{\mathrm{d} \sigma}=\frac{1}{\zeta_{3}^{3} s^{2}} \\
& \frac{\mathrm{d} \zeta_{1}}{\mathrm{~d} \sigma}=+s \sin \sigma\left(\mathbf{a}_{\mathrm{p}}^{*} \cdot \mathbf{i}_{\mathcal{L}}\right)+\left[\zeta_{1}+(1+s) \cos \sigma\right]\left(\mathbf{a}_{\mathrm{p}}^{*} \cdot \mathbf{j}_{\mathcal{L}}\right) \\
& \frac{\mathrm{d} \zeta_{2}}{\mathrm{~d} \sigma}=-s \cos \sigma\left(\mathbf{a}_{\mathrm{p}}^{*} \cdot \mathbf{i}_{\mathcal{L}}\right)+\left[\zeta_{2}+(1+s) \sin \sigma\right]\left(\mathbf{a}_{\mathrm{p}}^{*} \cdot \mathbf{j}_{\mathcal{L}}\right) \\
& \frac{\mathrm{d} \zeta_{3}}{\mathrm{~d} \sigma}=-\zeta_{3}\left(\mathbf{a}_{\mathrm{p}}^{*} \cdot \mathbf{j}_{\mathcal{L}}\right) \\
& \frac{\mathrm{d} \eta_{1}}{\mathrm{~d} \sigma}=\frac{\left(\mathbf{a}_{\mathrm{p}}^{*} \cdot \mathbf{k}_{\mathcal{L}}\right)}{2}\left(\eta_{4} \cos \sigma-\eta_{3} \sin \sigma\right) \\
& \frac{\mathrm{d} \eta_{2}}{\mathrm{~d} \sigma}=\frac{\left(\mathbf{a}_{\mathrm{p}}^{*} \cdot \mathbf{k}_{\mathcal{L}}\right)}{2}\left(\eta_{3} \cos \sigma+\eta_{4} \sin \sigma\right) \\
& \frac{\mathrm{d} \eta_{3}}{\mathrm{~d} \sigma}=\frac{\left(\mathbf{a}_{\mathrm{p}}^{*} \cdot \mathbf{k}_{\mathcal{L}}\right)}{2}\left(-\eta_{2} \cos \sigma+\eta_{1} \sin \sigma\right) \\
& \frac{\mathrm{d} \eta_{4}}{\mathrm{~d} \sigma}=\frac{\left(\mathbf{a}_{\mathrm{p}}^{*} \cdot \mathbf{k}_{\mathcal{L}}\right)}{2}\left(-\eta_{1} \cos \sigma-\eta_{2} \sin \sigma\right) .
\end{aligned}
$$

Eqs. (9)-(16) need to be integrated from the initial conditions

$\sigma=\sigma_{0}: \quad \tau=\tau_{0}, \quad \zeta_{1}=e_{0}, \quad \zeta_{2}=0, \quad \zeta_{3}=1 / h_{0}$, $\mathrm{n}=\mathrm{n}_{0}$.

\section{Singularities in Dromo}

There are two specific situations that make the integration of Dromo equations problematic:

Asymptotes:Eqs. (9)-(16) depend explicitly on the perturbing term $\mathbf{a}_{\mathrm{p}}^{*}=\mathbf{a}_{\mathrm{p}} /\left(\zeta_{3}^{4} s^{3}\right)$. These equations may become singular for $\left(\zeta_{3}^{4} s^{3}\right) \rightarrow 0$. The term $s=1+e \cos \theta$ becomes zero in two cases: (i) in the case of a parabolic orbit $(e=1)$, when the particle $O$ is sufficiently far from $C$, as $\theta \rightarrow \pi$. (ii) in the case of a hyperbolic orbit $(e>1)$, when the particle approaches the asymptote of the osculating hyperbola $\theta \rightarrow \arccos (-1 / e)$. In addition, if $\left(\zeta_{3}^{3} s^{2}\right)$ vanishes Eq. (9) becomes singular. For the case of a Keplerian orbit, where $h$ is constant, in the infinity it is $s \rightarrow 0$ although $h \neq 0$ at every step.

Angular momentum: If the position and velocity vectors are parallel, $\mathbf{r} \times \mathbf{v}=0$, the angular momentum vanishes and the departure perifocal frame $\mathcal{P}$ is undetermined. This problem may appear at departure if $\mathbf{r}_{0} \| \mathbf{v}_{0}$, or during the integration process due to external perturbations. As stated in Eq. (12), strong perturbations in the along-track direction have an important effect on the angular momentum of the particle. Both situations are intimately related with flyby trajectories. The first problem is a geometric issue that appears only in hyperbolic and parabolic orbits. The second problem relates to the magnitude and the effective direction of the perturbing term. In practice both phenomena are usually coupled.

The angular momentum appears recursively in the derivation of the equations of Dromo. The orbital plane is defined by means of the angular momentum vector.
The angular velocity of the perifocal departure frame $\mathcal{P}$, given in Eq. (5), becomes singular when the angular momentum becomes zero. Indeed, if the position and velocity vectors are parallel they do not define any plane, but a straight line. The direction of this line is given by the radius vector of the particle, which follows a rectilinear orbit along this line. The trajectory of the particle can be considered a degenerated conic section.

The description of the orbital plane adopted in Dromo is based on the dynamics of the rigid-body. Relying on the angular momentum of the particle a gyroscopic description of the orbital plane is provided. Quaternion $n$ is introduced to overcome the singularities associated to the usual Euler angles. But the gyroscopic approach is no longer valid when the equivalent rigid-body is no longer spinning, i.e. when the angular momentum vanishes.

Equation (12) shows the dependency of the angular momentum with respect to the perturbing terms. The angular momentum is affected by the component of the perturbing term along the direction of $\mathbf{j}_{\mathcal{L}}$. Numerical instabilities may be encountered when this particular projection of the perturbing acceleration grows. This is the typical case when the particle approaches a large planet (such as Jupiter) along an incoming trajectory that leads to a deep flyby.

\section{Modified Dromo equations}

Two alternative formulations (I and II) of Dromo are presented in this Section. Each formulation introduces a new set of Dromo elements, denoted by $\mathbf{D}_{\mathrm{I}}$ and $\mathbf{D}_{\mathrm{II}}$, respectively.

The first formulation eliminates all denominators from the right-hand side of the system of differential equations. To do so, an alternative definition of the fictitious time is provided. This introduces a slower time scale in the problem that may improve the accuracy of the integration. In addition, it may help to control the integrator step size more efficiently. The transformation from the $\mathbf{D}_{\mathrm{I}}$ elements to the state vector in Cartesian coordinates becomes singular for $s \rightarrow 0$ and the physical time becomes constant, even though there are no singularities in the evolution equations. This formulation may outperform the original Dromo numerically, but does not fully overcome the singularities.

The second formulation eliminates the discussed singularities in the strict sense, although it is singular for impact trajectories $r \rightarrow 0$. Neither $s$ nor the angular momentum appear in any denominator. However, as $s \rightarrow 0$ the right-hand side of the differential equation for the time goes to zero too. That is, although the formulation is not singular for $s \rightarrow 0$, the time becomes constant and the integration freezes in the limit case. It may behave better than Dromo as $s \rightarrow 0$ or $h \rightarrow 0$.

\subsection{Formulation I}

Consider the new set of variables $\left(\psi_{1}, \psi_{2}, \psi_{3}\right) \in \mathbb{R}^{3}$ defined as 
$\psi_{1}=1+\zeta_{1} \cos \sigma+\zeta_{2} \sin \sigma$,

$\psi_{2}=\zeta_{1} \sin \sigma-\zeta_{2} \cos \sigma, \quad \psi_{3}=a$.

The first variable, $\psi_{1}$, is equivalent to the term $s$ in Dromo. Variable $\psi_{2}$ appears naturally when deriving the expression for the velocity. Note that this pair of variables does not remain constant in the unperturbed case. The semimajor axis has been introduced because it is not affected by the $e \rightarrow 1$ singularity.

Remark. The variables $\psi_{1}, \psi_{2}$ are not constants in the unperturbed case.

The new set of variables $\mathbf{D}_{\mathbf{I}} \in \mathbb{R}^{7}$ is $\mathbf{D}_{\mathrm{I}}=\left(\psi_{1}, \psi_{2}, \psi_{3}, \eta_{1}\right.$, $\left.\eta_{2}, \eta_{3}, \eta_{4}\right)^{\top}$. There exists a bijective mapping between the original Dromo elements and the new variables, $f_{\mathrm{I}}: \mathbf{D} \mapsto \mathbf{D}_{\mathrm{I}}$ :

$\psi_{1}=1+\zeta_{1} \cos \sigma+\zeta_{2} \sin \sigma$

$\psi_{2}=\zeta_{1} \sin \sigma-\zeta_{2} \cos \sigma$

$\psi_{3}=\frac{1}{\zeta_{3}^{2}\left(1-\zeta_{1}^{2}-\zeta_{2}^{2}\right)}=\frac{1}{\zeta_{3}^{2} \ell}$

with the auxiliary term $\ell=1-\zeta_{1}^{2}-\zeta_{2}^{2}=2 \psi_{1}-\psi_{1}^{2}-\psi_{2}^{2}$. The inverse map $f_{\mathrm{I}}^{-1}: \mathbf{D}_{\mathrm{I}} \mapsto \mathrm{D}$ reads

$\zeta_{1}=-\cos \sigma+\psi_{1} \cos \sigma+\psi_{2} \sin \sigma$

$\zeta_{2}=-\sin \sigma+\psi_{1} \sin \sigma-\psi_{2} \cos \sigma$

$\zeta_{3}=\frac{1}{\sqrt{\psi_{3}\left(2 \psi_{1}-\psi_{1}^{2}-\psi_{2}^{2}\right)}}=\frac{1}{\sqrt{\psi_{3} \ell}}$.

Under this new formulation the position and velocity vectors of the particle result in

$\mathbf{r}=\frac{\psi_{3} \ell}{\psi_{1}} \mathbf{i}_{\mathcal{L}}, \quad \mathbf{v}=\frac{1}{\psi_{3} \ell}\left(\psi_{2} \mathbf{i}_{\mathcal{L}}+\psi_{1} \mathbf{i}_{\mathcal{L}}\right)$.

The Keplerian energy of the particle is given by

$\mathcal{E}=\frac{v^{2}}{2}-\frac{1}{r}=-\frac{1}{2 \psi_{3}}$.

The derivative of the energy w.r.t. the ideal anomaly, $\sigma$, is

$\frac{\mathrm{d} \mathcal{E}}{\mathrm{d} \sigma}=\frac{1}{2 \psi_{3}^{2}} \frac{\mathrm{d} \psi_{3}}{\mathrm{~d} \sigma}=\frac{\mathrm{d} r}{\mathrm{~d} \sigma}\left(\mathbf{a}_{\mathrm{p}} \cdot \mathbf{i}_{\mathcal{L}}\right)+r\left(\mathbf{a}_{\mathrm{p}} \cdot \mathbf{j}_{\mathcal{L}}\right)$

This expression leads to the evolution of $\psi_{3}$

$\frac{\mathrm{d} \psi_{3}}{\mathrm{~d} \sigma}=\frac{2 \psi_{3}^{3} \ell}{\psi_{1}^{2}}\left[\psi_{2}\left(\mathbf{a}_{\mathrm{p}} \cdot \mathbf{i}_{\mathcal{L}}\right)+\psi_{1}\left(\mathbf{a}_{\mathrm{p}} \cdot \mathbf{j}_{\mathcal{L}}\right)\right]$

Note that the problem is still singular for $\psi_{1}=0$. A complete regularization of the equations requires not only the dependent variables to be changed, but also the independent variable.

Let $\chi$ be the new independent variable, related to the ideal anomaly through

$\frac{\mathrm{d} \sigma}{\mathrm{d} \chi}=\psi_{1}^{3}$
This new fictitious time may lead to a more accurate integration of the flyby phase.

Dromo equations become:

$$
\begin{aligned}
& \frac{\mathrm{d} \tau}{\mathrm{d} \chi}=\left(\psi_{3} \ell\right)^{3 / 2} \psi_{1} \\
& \frac{\mathrm{d} \psi_{1}}{\mathrm{~d} \chi}=-\psi_{2} \psi_{1}^{3}+2\left(\psi_{3} \ell\right)^{2} \psi_{1}\left(\mathbf{a}_{\mathrm{p}} \cdot \mathbf{j}_{\mathcal{L}}\right) \\
& \frac{\mathrm{d} \psi_{2}}{\mathrm{~d} \chi}=\left(\psi_{1}-1\right) \psi_{1}^{3}+\left(\psi_{3} \ell\right)^{2}\left[\psi_{1}\left(\mathbf{a}_{\mathrm{p}} \cdot \mathbf{i}_{\mathcal{L}}\right)+\psi_{2}\left(\mathbf{a}_{\mathrm{p}} \cdot \mathbf{j}_{\mathcal{L}}\right)\right] \\
& \frac{\mathrm{d} \psi_{3}}{\mathrm{~d} \chi}=2 \psi_{3}^{3} \psi_{1} \ell\left[\psi_{2}\left(\mathbf{a}_{\mathrm{p}} \cdot \mathbf{i}_{\mathcal{L}}\right)+\psi_{1}\left(\mathbf{a}_{\mathrm{p}} \cdot \mathbf{j}_{\mathcal{L}}\right)\right] \\
& \frac{\mathrm{d} \eta_{1}}{\mathrm{~d} \chi}=\frac{\left(\psi_{1} \ell\right)^{2}}{2}\left(\eta_{4} \cos \sigma-\eta_{3} \sin \sigma\right)\left(\mathbf{a}_{\mathrm{p}} \cdot \mathbf{k}_{\mathcal{L}}\right) \\
& \frac{\mathrm{d} \eta_{2}}{\mathrm{~d} \chi}=\frac{\left(\psi_{1} \ell\right)^{2}}{2}\left(\eta_{3} \cos \sigma+\eta_{4} \sin \sigma\right)\left(\mathbf{a}_{\mathrm{p}} \cdot \mathbf{k}_{\mathcal{L}}\right) \\
& \frac{\mathrm{d} \eta_{3}}{\mathrm{~d} \chi}=\frac{\left(\psi_{1} \ell\right)^{2}}{2}\left(-\eta_{2} \cos \sigma+\eta_{1} \sin \sigma\right)\left(\mathbf{a}_{\mathrm{p}} \cdot \mathbf{k}_{\mathcal{L}}\right) \\
& \frac{\mathrm{d} \eta_{4}}{\mathrm{~d} \chi}=\frac{\left(\psi_{1} \ell\right)^{2}}{2}\left(-\eta_{1} \cos \sigma-\eta_{2} \sin \sigma\right)\left(\mathbf{a}_{\mathrm{p}} \cdot \mathbf{k}_{\mathcal{L}}\right) .
\end{aligned}
$$

Eqs. (17)-(25) define the new system of differential equations that govern the evolution of the Dromo elements.

The number of equations to be integrated has been increased from eight to nine, because the ideal anomaly $\sigma$ is no longer the independent variable and is integrated from Eq. (17). The initial conditions are defined by the value of the parameters at epoch $\chi=0 \quad\left(\sigma=\sigma_{0}\right)$, corresponding to

$\chi=0: \quad \sigma=\sigma_{0}, \tau=0, \psi_{1}=1+e_{0} \cos \sigma_{0}, \psi_{2}=e_{0} \sin \sigma_{0}$.

This formulation is free of singularities in the strict sense. However, as $\psi_{1} \rightarrow 0$ the right-hand side of the equations vanishes and the integration freezes. In addition, the velocity may encounter a singularity as the term $\psi_{3} \ell$ vanishes: note that it is $\ell=1-e^{2}$, which goes to zero as $e \rightarrow 1$.

\subsection{Formulation II}

Let $\left(\varphi_{1}, \varphi_{2}, \varphi_{3}\right) \in \mathbb{R}^{3}$ define the triple

$\varphi_{1}=\zeta_{1}, \quad \varphi_{2}=\zeta_{2}, \quad \varphi_{3}=\frac{r^{2}}{2}=\frac{1}{2 \zeta_{3}^{4} s^{2}}$.

The first two terms are equivalent to the first elements in $\mathbf{D}$, whereas the third term is proportional to the distance to the center of attraction.

Remark. Variable $\varphi_{3}$ is not an element.

The new set of variables $\mathbf{D}_{\mathrm{II}} \in \mathbb{R}^{7}$ is of the form $\mathbf{D}_{\mathrm{II}}=\left(\varphi_{1}, \varphi_{2}, \varphi_{3}, \eta_{1}, \eta_{2}, \eta_{3}, \eta_{4}\right)^{\top}$. In this case the map $\mathbf{D} \mapsto \mathbf{D}_{\mathrm{II}}$ is already established by the definition of $\mathbf{D}_{\mathrm{II}}$. The inverse mapping only requires to compute

$\zeta_{3}=\frac{1}{\sqrt[4]{2 s^{2} \varphi_{3}}}$. 
Let $\Upsilon$ be the new independent variable, related to the ideal anomaly through

$\frac{\mathrm{d} \sigma}{\mathrm{d} \Upsilon}=\frac{s}{r^{2}}=\frac{s}{2 \varphi_{3}}=\zeta_{3}^{4} s^{3}$.

Under this new formulation, the evolution equations become

$\frac{\mathrm{d} \tau}{\mathrm{d} \Upsilon}=\sqrt{\frac{s}{\sqrt{2 \varphi_{3}}}}$

$\frac{\mathrm{d} \varphi_{1}}{\mathrm{~d} \Upsilon}=+s \sin \sigma\left(\mathbf{a}_{\mathrm{p}} \cdot \mathbf{i}_{\mathcal{L}}\right)+\left[\varphi_{1}+(1+s) \cos \sigma\right]\left(\mathbf{a}_{\mathrm{p}} \cdot \mathbf{j}_{\mathcal{L}}\right)$

$\frac{\mathrm{d} \varphi_{2}}{\mathrm{~d} \Upsilon}=-s \cos \sigma\left(\mathbf{a}_{\mathrm{p}} \cdot \mathbf{i}_{\mathcal{L}}\right)+\left[\varphi_{2}+(1+s) \sin \sigma\right]\left(\mathbf{a}_{\mathrm{p}} \cdot \mathbf{j}_{\mathcal{L}}\right)$

$\frac{\mathrm{d} \varphi_{3}}{\mathrm{~d} \Upsilon}=\varphi_{1} \sin \sigma-\varphi_{2} \sin \sigma$

$\frac{\mathrm{d} \eta_{1}}{\mathrm{~d} \Upsilon}=\frac{\left(\mathbf{a}_{\mathrm{p}} \cdot \mathbf{k}_{\mathcal{L}}\right)}{2}\left(\eta_{4} \cos \sigma-\eta_{3} \sin \sigma\right)$

$\frac{\mathrm{d} \eta_{2}}{\mathrm{~d} \Upsilon}=\frac{\left(\mathbf{a}_{\mathrm{p}} \cdot \mathbf{k}_{\mathcal{L}}\right)}{2}\left(\eta_{3} \cos \sigma+\eta_{4} \sin \sigma\right)$

$\frac{\mathrm{d} \eta_{3}}{\mathrm{~d} \Upsilon}=\frac{\left(\mathbf{a}_{\mathrm{p}} \cdot \mathbf{k}_{\mathcal{L}}\right)}{2}\left(-\eta_{2} \cos \sigma+\eta_{1} \sin \sigma\right)$

$\frac{\mathrm{d} \eta_{4}}{\mathrm{~d} \Upsilon}=\frac{\left(\mathbf{a}_{\mathrm{p}} \cdot \mathbf{k}_{\mathcal{L}}\right)}{2}\left(-\eta_{1} \cos \sigma-\eta_{2} \sin \sigma\right)$.

These equations may become singular as $\varphi_{3} \rightarrow 0$, i.e. $r \rightarrow 0$. The equations are not fully regularized, but the singularity for $s \rightarrow 0$ is overcome. The state vector is defined by

$\mathbf{r}=\sqrt{2 \varphi_{3}}\left(\cos \sigma \mathbf{i}_{\mathcal{P}}+\sin \sigma \mathbf{j}_{\mathcal{\rho}}\right)$,

$\mathbf{v}=\sqrt{\frac{1}{s \sqrt{2 \varphi_{3}}}}\left[-\left(\varphi_{2}+\sin \sigma\right) \mathbf{i}_{\varphi}+\left(\varphi_{1}+\cos \sigma\right) \mathbf{j}_{\mathcal{P}}\right]$.

\section{Numerical experiments}

The numerical performance of Dromo may be affected by rapid changes in the angular momentum. In order to test the formulation in actual applications the impact of NEAs against the Earth are addressed, where trajectories may be close to the discussed limit cases. The first considered asteroid is $2008 \mathrm{TC}_{3}$, which impacted the Earth in 2008-Oct-07. Jenniskens et al. (2009) provide a concise overview of the impact and the asteroid itself. According to the JPL/NEOP ${ }^{1}$ database, asteroids $2014 \mathrm{AA}$ and $2014 \mathrm{LY}_{21}$ yield the minimum close approach distance in 2014. They are included in the analysis for this reason. Table 1 summarizes the main properties of the discussed asteroids. The minimum angular momentum associated to these asteroids is normalized using $\ell_{\mathrm{c}}=a_{\min }$ and $t_{\mathrm{c}}=\left(\ell_{\mathrm{c}}^{3} / \mu\right)^{1 / 2}$, providing the maximum value of $\zeta_{3} \in \mathbf{D}$. Dromo variable $\zeta_{3}$ does not grow rapidly in these cases.

\footnotetext{
${ }^{1}<$ http://neo.jpl.nasa.gov/>.
}

The orbits of the three asteroids from Table 1 are integrated using Dromo. Integration starts when the asteroids enter the sphere of influence of the Earth, considering an approximate value of $10^{6} \mathrm{~km}$. The force model includes the major planets, Pluto, the Moon, and the four major asteroids (Ceres, Vesta, Pallas, Hygiea), relying on the DE432 ephemeris. A $100 \times 100$ gravity model is considered, using the coefficients from GGM03S. Relativistic corrections include the lense-thirring effect, and the influence of the Earth oblateness. The GCRS/ITRS conversion is conducted by means of the CIO (celestial intermediate origin) based transformation. Corrections from the Earth Orientation Parameters comply with the IAU06 standards. Figure 2 shows the error in position when the trajectory integrated with Dromo is compared against the solution from JPL/Horizons. The error in position $\varepsilon(\tau)$ is measured as

$\varepsilon(\tau)=\left\|\mathbf{r}(\tau)-\mathbf{r}_{\text {ref }}(\tau)\right\|$,

where $\mathbf{r}$ denotes the integrated solution and $\mathbf{r}_{\mathrm{ref}}$ is the solution from JPL/Horizons. Dromo encounters no numerical issues when integrating these orbits. The error for asteroids $2014 \mathrm{AA}$ and $2014 \mathrm{LY}_{21}$ remains under $1 \mathrm{~m}$ during the entire integration, except for a slight peak about the time of closest approach in the orbit of asteroid $2014 \mathrm{LY}_{21}$. The final error in this case is about $2.5 \mathrm{~m}$, even though the mean error in position is $0.31 \mathrm{~m}$. The average of the error in position for asteroid $2014 \mathrm{AA}$ is $0.34 \mathrm{~m}$, being its diameter about $2.5 \mathrm{~m}$. The error in determining the orbit of asteroid $2008 \mathrm{TC}_{3}$ grows faster during the last day of the integration, being the estimated diameter of the asteroid $4.1 \mathrm{~m}$. The discrepancy is believed to be caused by the limitations of the force model and differences in the dynamical system. In addition, the orbit determined by Horizons may include observational data.

No numerical issues have been encountered when propagating this sample. In order to study the behavior of Dromo in the vicinity of the singularity a set of theoretical test cases is proposed in the following.

\subsection{Isolating the singularity}

The singularity in Dromo that has been addressed appears under very specific conditions. Among the different scenarios on which $s \rightarrow 0$, deep flybys are considered. When the particle approaches the sphere of influence of the attracting body it may enter the sphere close to the asymptote along a hyperbolic orbit. The incoming velocity vector would be quasi-parallel to the radius-vector, leading to the discussed singularities. As the minimum flyby distance decreases the perturbation of the third body increases, with a direct impact on the angular momentum. A number of theoretical flybys about the Earth and Jupiter are designed to test the performance of Dromo and the new versions Dromo-I and Dromo-II.

The designed experiment consists in propagating the heliocentric orbit of a virtual asteroid subject to the 
Table 1

Orbital parameters since entrance in the sphere of influence of the Earth to the close approach. (Source: JPL/Horizons).

\begin{tabular}{|c|c|c|c|c|c|}
\hline Asteroid & Closest approach & $e_{\min }$ & $h_{\min }\left[\mathrm{km}^{2} \mathrm{~s}^{-1}\right]$ & $\zeta_{3, \max }$ & $\overline{\text { Diameter }[\mathrm{m}]}$ \\
\hline $2008 \mathrm{TC}_{3}$ & 2008-Oct-07 02:46 UTC & 1.5293 & $9.867 \times 10^{3}$ & 0.86 & $4.1 \pm 0.3^{1}$ \\
\hline $2014 \mathrm{AA}$ & 2014-Jan-02 04:02 UTC & 1.0313 & $1.602 \times 10^{4}$ & 3.93 & $2.5 \pm 0.5^{2}$ \\
\hline $2014 \mathrm{LY}_{21}$ & 2014-Jun-03 22:38 UTC & 4.2909 & $5.065 \times 10^{3}$ & 0.24 & $6.0 \pm 2.0^{3}$ \\
\hline
\end{tabular}

${ }^{1}$ Jenniskens et al. (2009).

2 Chesley et al. (2014).

${ }^{3}$ MPEC 2014-L48: $2014 \mathrm{LY}_{21}$.

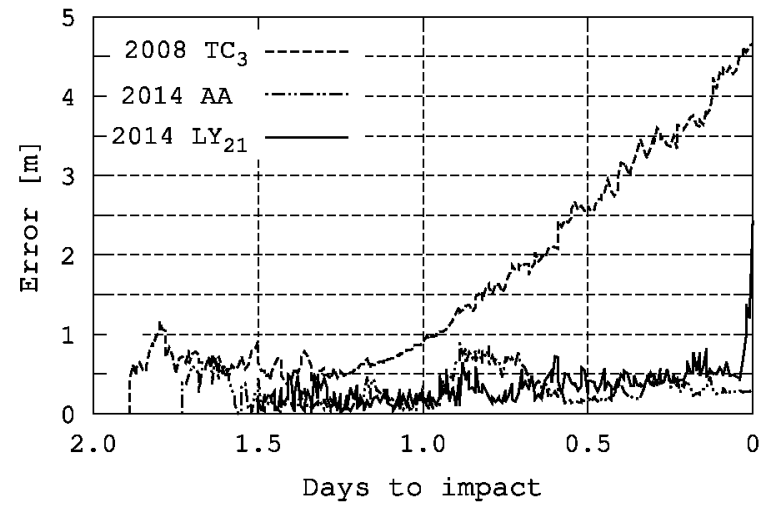

Fig. 2. Validation of Dromo formulation in propagating asteroid close approaches.

gravitational attraction of a certain planet. The model is reduced to the circular restricted three-body problem (CRTBP) in order to gain full control on the perturbations and dynamics of the encounter. The orbit of the asteroid intersects the orbit of the planet and performs a planetocentric flyby. There are two possible approaches to the problem. On the one hand, the perturbed heliocentric orbit of the asteroid can be integrated directly. On the other hand, the orbit can be divided in three arcs: (i) the initial heliocentric arc until the asteroid reaches the sphere of influence of the planet, (ii) the planetocentric flyby inside the sphere of influence of the planet (perturbed by the Sun), (iii) the final heliocentric arc once the asteroid has left the sphere of influence of the planet. This second approach requires to switch the primary attracting body when entering and leaving the sphere of influence. Figure 3 depicts the geometry of the problem.

Let $\mathcal{C}$ denote the primary attractive center, let $\mathcal{S}$ denote the secondary and let $O$ be the asteroid. Neglecting the mass of the asteroid $\left(m_{O} \ll m_{\mathcal{C}}, m_{\mathcal{S}}\right)$ the restricted threebody problem is governed by the equation

$\frac{\mathrm{d}^{2} \mathbf{r}_{\mathcal{O}}}{\mathrm{d} \tau^{2}}+\mu_{C} \frac{\mathbf{r}_{C O}}{r_{C O}^{3}}=-\mu_{\mathcal{S}}\left(\frac{\mathbf{r}_{\mathcal{S O}}}{r_{S O}^{3}}-\frac{\mathbf{r}_{\mathcal{S O}}}{r_{S C}^{3}}\right)=\mathbf{a}_{\mathrm{p}}$,

where vector $\mathbf{r}_{i j}$ reads $\mathbf{r}_{i j}=\mathbf{r}_{j}-\mathbf{r}_{i}$ for $i, j=\{C, \mathcal{S}, O\}$ and $r_{i j}=\left\|\mathbf{r}_{i j}\right\|$. The perturbing acceleration is then written:

$\mathbf{a}_{\mathrm{p}}=-\mu_{\mathcal{S}}\left(\frac{\mathbf{r}_{\mathcal{S O}}}{r_{\mathcal{S O}}^{3}}-\frac{\mathbf{r}_{\mathcal{S C}}}{r_{\mathcal{S C}}^{3}}\right)=-\mu_{\mathcal{S}}\left[\frac{\mathbf{r}_{\mathcal{S O}}}{r_{\mathcal{S C}}^{3}}\left(\frac{r_{\mathcal{S C}}^{3}}{r_{\mathcal{S O}}^{3}}-1\right)-\frac{\mathbf{r}_{O C}}{r_{\mathcal{S C}}^{3}}\right]$.

Let $f$ denote the term

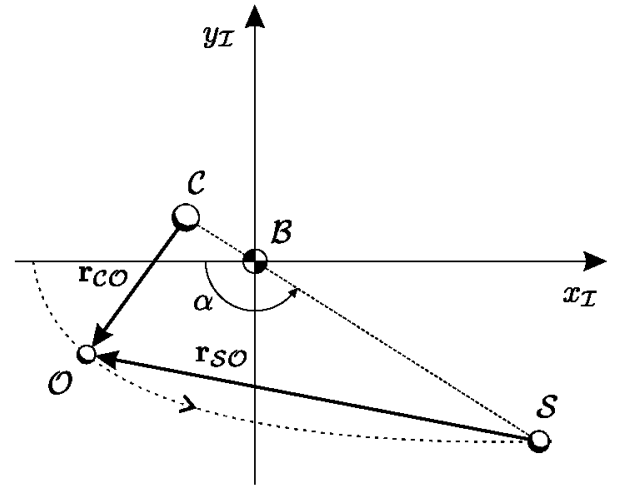

Fig. 3. Geometry of the three-body problem. The primary body is $\mathcal{C}, \mathcal{S}$ denotes the secondary body, $O$ denotes the orbiting particle and $\mathcal{B}$ is the barycenter of the system.

$f=\frac{r_{\mathcal{S C}}^{3}}{r_{\mathcal{S O}}^{3}}-1$

When $r_{C S} \sim r_{S O}$ the terms to subtract are similar and the computation of $f$ may lead to the numerical catastrophic cancellation. The term $f$ admits an alternative expression considering the relation

$\frac{r_{S C}^{2}}{r_{S O}^{2}}=\frac{\left(\mathbf{r}_{S O}+\mathbf{r}_{O C}\right) \cdot\left(\mathbf{r}_{S O}+\mathbf{r}_{O C}\right)}{\mathbf{r}_{S O} \cdot \mathbf{r}_{S O}}=1+\Lambda$,

and having introduced the auxiliary term:

$\Lambda=\frac{\mathbf{r}_{O C} \cdot\left(\mathbf{r}_{O C}+2 \mathbf{r}_{S O}\right)}{\mathbf{r}_{S O} \cdot \mathbf{r}_{\mathcal{S O}}}$.

The term $f=f(\Lambda)$ reduces to

$f(\Lambda)=\Lambda \frac{3+3 \Lambda+\Lambda^{2}}{(1+\Lambda)^{3 / 2}+1}$,

which requires no numerical subtractions. The perturbation from the third body becomes

$\mathbf{a}_{\mathrm{p}}=-\mu_{\mathcal{S}}\left[\frac{\mathbf{r}_{\mathcal{S O}}}{r_{\mathcal{S C}}^{3}} f(\Lambda)-\frac{\mathbf{r}_{O C}}{r_{S C}^{3}}\right]$.

In the CRTBP the secondary body is assumed to follow a circular orbit about the primary body. Under this assumption the synodic rate $\omega_{\mathrm{s}}$ is constant,

$\omega_{\mathrm{s}}=\sqrt{\frac{\mu_{\mathcal{C}}+\mu_{S}}{r_{S C}^{3}}}$. 
Equations are normalized so as $\mu_{C}=1$ and $r_{\mathcal{S C}}=1$. The dynamical equations in the CRTBP admit a first integral of motion, namely the Jacobi integral. If $v$ denotes the velocity of the particle in the synodic frame, $\mathcal{J}$ is the Jacobi constant and $V$ is the potential, the Jacobi integral reads

$v^{2}=-\mathcal{T}-2 V$.

Let $\left(x_{\mathrm{s}}, y_{\mathrm{s}}, z_{\mathrm{s}}\right)^{\top} \in \mathbb{R}^{3}$ be the barycentric coordinates of a particle in the synodic rotating reference frame. The Jacobi constant is given by

$\mathcal{J}=x_{\mathrm{s}}^{2}+y_{\mathrm{s}}^{2}-\left(\dot{x}_{\mathrm{s}}^{2}+\dot{y}_{\mathrm{s}}^{2}+\dot{z}_{\mathrm{s}}^{2}\right)+\frac{2 \varrho}{r_{S O}}+\frac{2(1-\varrho)}{r_{C O}}$.

where $\varrho=m_{\mathcal{S}} /\left(m_{\mathcal{C}}+m_{\mathcal{S}}\right)$ is the mass ratio. How the Jacobi constant is preserved during the integration indicates the accuracy and stability of the formulation. The orbit of the asteroid is propagated for one complete revolution about the Sun: the final value of the Jacobi constant, $\mathcal{J}_{\mathrm{f}}$, is then compared to the initial value, $\mathcal{J}_{0}$, and the relative error in preserving the Jacobi constant is computed

Error $=\frac{\left|\mathcal{J}_{\mathrm{f}}-\mathcal{J}_{0}\right|}{\mathcal{J}_{0}}=\frac{\Delta \mathcal{J}}{\mathcal{J}_{0}}$.

Four different flyby scenarios are designed, as summarized in Table 2. In the first two cases the asteroid encounters the Earth, whereas in the third and fourth cases the perturbing planet is Jupiter. Each flyby is defined by the pericenter height $\left(d_{\text {per }}\right)$ and the eccentricity of the planetocentric orbit when entering the sphere of influence of the planet $\left(e_{\infty}\right)$. The direction of the asymptote $\left(\theta_{\text {asy }}\right)$ can be compared to the initial true anomaly on the planetocentric orbit $\left(\theta_{\infty}\right)$. Note that Case 3 represents a direct impact against Jupiter along a quasi-parabolic orbit. Case 4 is a moderate version of Case 3 , where the asteroid is initially very close to the asymptote. Both cases capture the discussed singularities of Dromo. Table 2 also presents the net $\Delta V$ achieved by the asteroid during the flyby. The heliocentric orbit is initially determined by the initial eccentricity $e_{0}$. The orbital encounter occurs at $\alpha=\alpha_{\text {enc }}$ (see Fig. 3), being the encounter the instant of the closest approach to the planet.

The performance of the original Dromo scheme (Peláez et al., 2007; Urrutxua et al., 2013) is compared against the new versions Dromo-I and Dromo-II. Cowell's method, the solution from the Kustaanheimo-Stiefel transformation (Kustaanheimo and Stiefel, 1965), and the Sperling-Burdet regularization (Burdet, 1968) are integrated as reference solutions. These formulations are compared not only in terms of the error in preserving the Jacobi constant, but also regarding the CPU runtime on an Intel Core i7$4770 @ 3.40 \mathrm{GHz}$ machine. The problem is solved fifteen consecutive times and the runtime is the total cumulative time, divided by fifteen. This technique averages out possible instabilities in the integration. The problem is integrated using a Runge-Kutta-Fehlberg 7(8) integrator and a Dormand-Prince 8(53) integrator, in double precision. Both the direct integration of the problem (where the Sun is always the primary body $\mathcal{C}$ ) and the solution where the primary is switched when the asteroid is inside the sphere of influence of the planet (the primary body is now the planet, and the Sun is the secondary) are computed.

\subsection{Case 1}

Figures 4-8 display the the CPU runtime and the relative error in preserving the Jacobi constant for each of the four cases defined in Table 2. Each point in the figures corresponds to a different integration tolerance, which varies from $10^{-5}$ to $10^{-15}$. Figure 4 corresponds to Case 1 . It is observed that the $\mathrm{KS}$ and the $\mathrm{SB}$ regularizations exhibit similar performances. The three versions of Dromo are the most efficient when integrating the direct problem with a RKF7(8) schema. When integrating the problem with DoPri8(53) Dromo-I and Dromo-II become the fastest formulations. Switching the primaries benefits KS and SB formulations, which are only outperformed by Dromo-I.

\subsection{Case 2}

Case 2 defines a closer flyby about the Earth. Dromo-II is the preferred formulation for integrating the direct problem with a RKF7(8) scheme. Dromo-I shows clear improvements in the computational efficiency when the primaries are switched, for both $\operatorname{RKF7(8)}$ and DoPri8(53) integrators. When the problem is integrated as one single are and using DoPri8(53), the SB regularization stands out as the fastest method.

\subsection{Case 3}

Case 3 is a limit case specifically conceived for finding the singularity in Dromo. The direct integration of the

Table 2

Definition of the numerical test cases

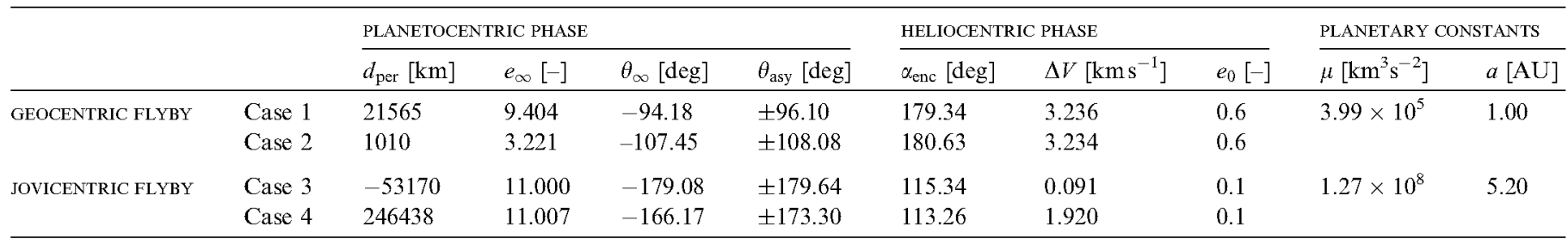




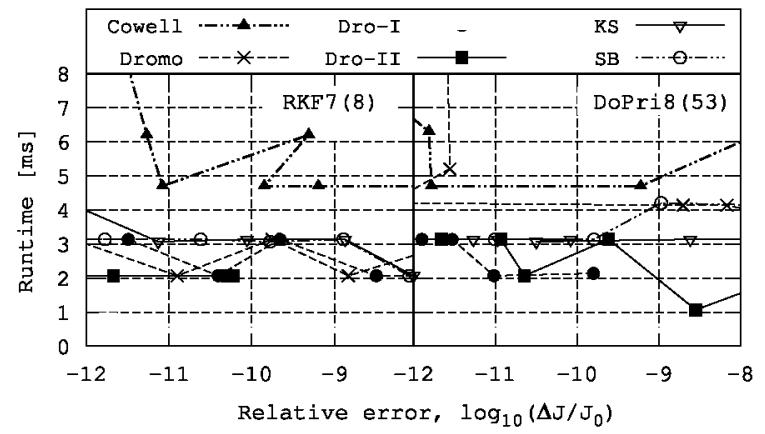

(a) Direct integration

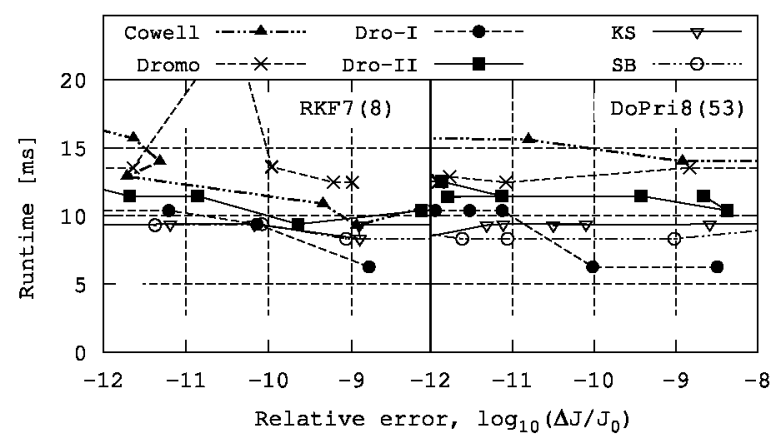

(b) Switched integration

Fig. 4. Performance comparison for Case 1.

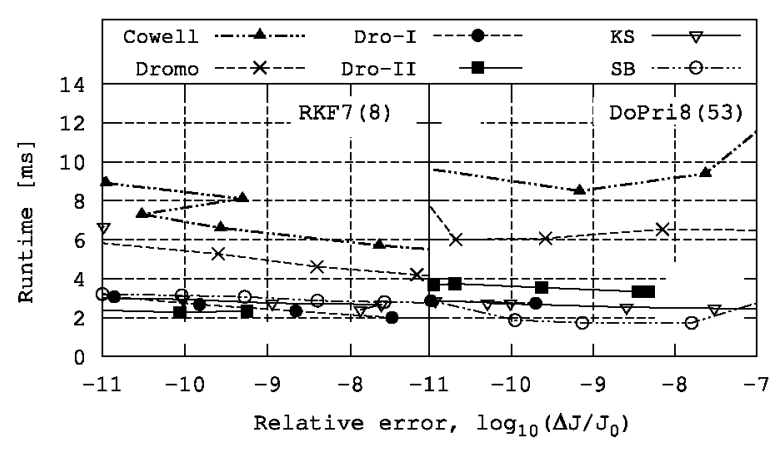

(a) Direct integration

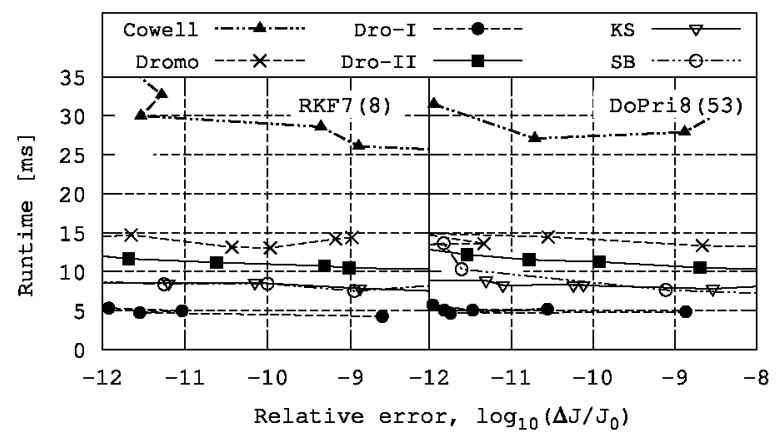

(b) Switched integration

Fig. 5. Performance comparison for Case 2

problem fails when the formulation becomes singular. The numerical issues encountered by Dromo can be analyzed in Fig. 6. As the asteroid approaches Jupiter the term $\zeta_{3}$

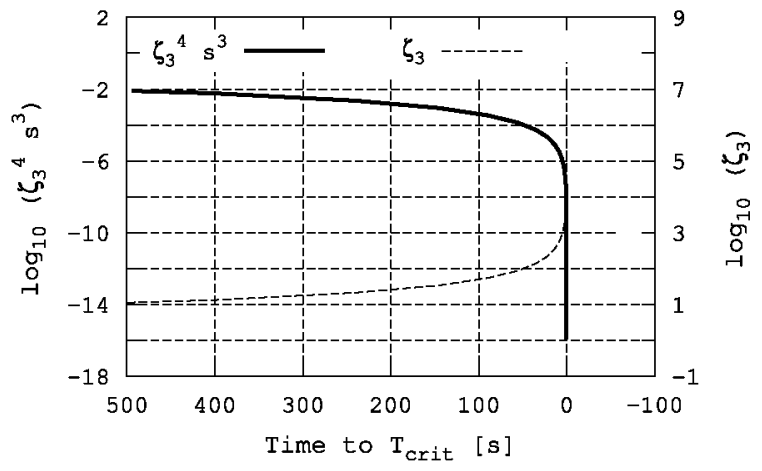

Fig. 6. Evolution of the terms $\left(\zeta_{3}^{4} s^{3}\right)$ and $\zeta_{3}$ in Case 3 .

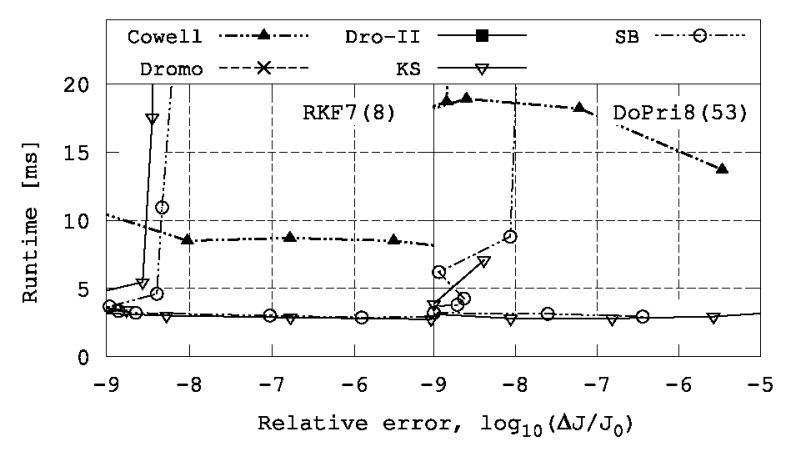

(a) Direct integration

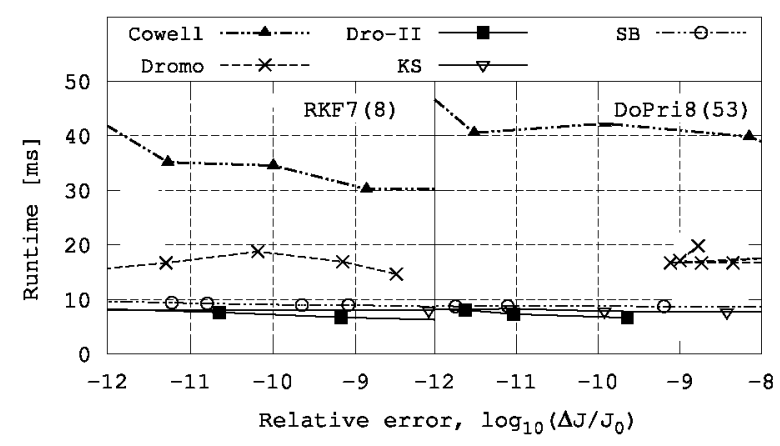

(b) Switched integration

Fig. 7. Performance comparison for Case 3.

grows rapidly, $\zeta_{3} \rightarrow 10^{8}$, since the angular momentum goes to zero. Despite the growth of $\zeta_{3}$, the denominator $\left(\zeta_{3}^{4} s^{3}\right)$ that defines the term $\mathbf{a}_{\mathrm{p}}^{*}=\mathbf{a}_{\mathrm{p}} /\left(\zeta_{3}^{4} s^{3}\right)$ in Eqs. (9)-(16) becomes zero. During the integration it is observed that the product $\left(\zeta_{3}^{3} s^{2}\right)$ goes to zero too. Hence, the transformation in Eq. (6) becomes singular at that point. This is caused by the fact that $s \rightarrow 0$ compensates the growth rate of $\zeta_{3}$. This phenomenon makes the equations of Dromo singular. The singularity is found at $T_{\text {crit }}=1100.7197$ days. The singularity appears when the position and velocity vectors become parallel due to the perturbation from the third body.

The fact that the term $\left(\zeta_{3}^{4} s^{3}\right)$ goes to zero has additional consequences. In Dromo-II the derivative of the ideal anomaly with respect to the new independent variable $\Upsilon$ 


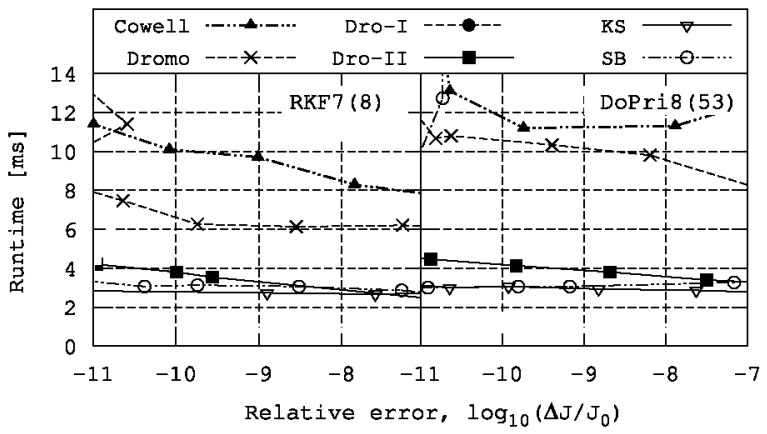

(a) Direct integration

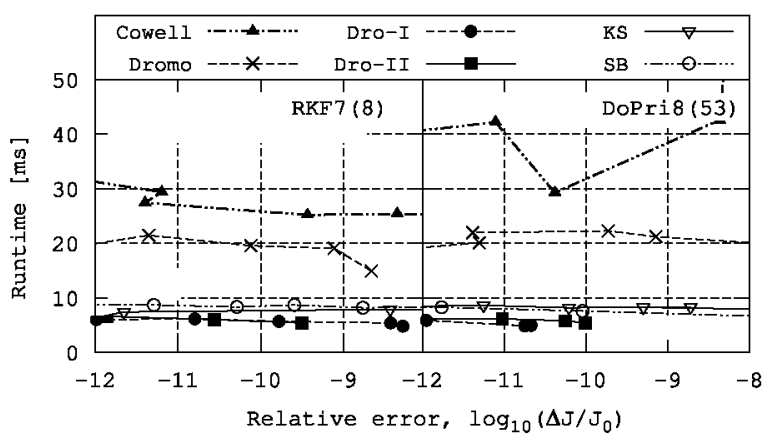

(b) Switched integration

Fig. 8. Performance comparison for Case 4.

Eq. (17)- is precisely $\left(\zeta_{3}^{4} s^{3}\right)$. Hence, the ideal anomaly becomes constant and the formulation freezes. The same issue is detected when integrating Dromo-I.

The performance of different formulations when integrating this critical case is shown in Fig. 7. It is observed that the direct integration of the problem using any version of Dromo leads to extremely long computing times which are out of scale, as discussed in the previous lines. The KS transformation slightly outperforms Cowell's method and the SB formulation. When the primaries are switched the combinations of DoPri8(53)/ Dromo-II and RKF7(8)/Dromo-II become the most efficient methods to integrate. The results obtained with Dromo-I are omitted from Fig. 7b since they correspond to extremely long computational times.

\subsection{Case 4}

Case 4 is still a deep flyby, but it is possible to integrate the problem with Dromo and Dromo-II. When considering the direct integration of the problem only Dromo-II is able to match the performance of the KS and SB formulations. Dromo-I is problematic to integrate in this case, the resulting computational times are completely out of scale and the results are not included in Fig. 8a. However, if the primaries are switched the two new versions of Dromo stand out as the fastest formulations. The CPU runtime corresponding to the original Dromo remains over that for the KS, SB, Dromo-I and Dromo-II.

\section{Conclusions}

The performance of the Dromo orbital propagator depends upon the behavior of the angular momentum. As proven in the present work, this phenomenon is the main source of instabilities in Dromo. Not only variable $\zeta_{3}$ may grow rapidly, but also the gyroscopic description of the orbital plane is no longer valid when the angular momentum of the particle vanishes. Numerical instabilities may be encountered during the integration if the angular momentum is strongly affected by perturbations. The integration of the actual orbit of three different NEAs yields an error that is comparable or even below the diameter of the asteroids, when compared against the solution from JPL/Horizons. This experiment proves that Dromo is applicable to determining the orbit of real bodies conducting close approaches. The main question is how close to the singularity real applications may be.

Two alternative reformulations of Dromo have been provided to mitigate the effects of the singularities. Dromo-I proposes a new definition of the independent variable, in an attempt to improve the integrator performance in the vicinity of the singularity. Although it eliminates the singularities from the system of differential equations, the transformation to the actual state vector depends on the angular momentum and becomes singular in the limit case $h \rightarrow 0$. Dromo-II overcomes the singularities in Dromo, at the price of becoming singular in the case of a direct impact $r \rightarrow 0$. In addition, the physical time may become constant when integrating both formulations, leading to long computational times.

Numerical results confirm that Dromo may outperform other formulations in weakly perturbed environments. As the flyby becomes closer to a parabolic impact trajectory the performance of Dromo is depreciated, and Dromo-I/ II exhibit important improvements in performance. Although Dromo-I may be the most efficient numerically, in the limit case Dromo-II stands out as the fastest formulation.

Despite becoming singular in the theoretical limit, Dromo still behaves well in the proximity of the asymptotes of a hyperbola, or when the decrement in the angular momentum is not sufficiently violent. That is, the domain where the equations are ill-conditioned does not extend far from the singularity. When the angular momentum is sufficiently large Dromo may outperform equivalent formulations. Numerical issues have been detected when integrating directly a highly perturbed theoretical orbital encounter, without switching the primary attracting body inside the sphere of influence of the planet. Switching the primary attracting body partially mitigates the integration issues.

Future work includes a detailed analysis of the practical situations where the propagation may be affected by this sort of singularities. Different surveys of comets, asteroids, and spacecraft should be conducted. Dromo encountered 
no numerical issues when propagating the example asteroids, but its behavior may depend on the application.

\section{Acknowledgments}

This work was carried out within the framework of the research project entitled Dynamic Simulation of Space Complex Systems (ESP2013 41634-P) supported by the Spanish Ministry of Economy and Competitiveness. Authors thank Spanish Government for its support, and $H$. Urrutxua for his help with the numerical integration. J. Roa especially thanks "La Caixa" for his doctoral fellowship.

\section{References}

Acedo, L., 2014. The flyby anomaly: A case for strong gravitomagnetism? Adv. Space Res. 54, 788-796.

Adler, S.L., 2009. Can the flyby anomaly be attributed to earth-bound dark matter? Phys. Rev. D. 79, 023505.

Anderson, J.D., Campbell, J.K., Ekelund, J.E., Ellis, J., Jordan, J.F., 2008. Anomalous orbital-energy changes observed during spacecraft flybys of earth. Phys. Rev. Lett. 100, 091102.

Baù, G., Bombardelli, C., 2014. Time elements for enhanced performance of the Dromo orbit propagator. Astron. J. 148, 43.

Baù, G., Urrutxua, H., Peláez, J., 2014. EDROMO: an accurate propagator for elliptical orbits in the perturbed two-body problem. Adv. Astronaut. Sci. 152, 379-399.

Bombardelli, C., Baù, G., Peláez, J., 2011. Asymptotic solution for the two-body problem with constant tangential thrust acceleration. Celestial Mech. Dyn. Astron. 110, 239-256.

Bond, V.R., 1982. Error propagation in the numerical solutions of the differential equations of orbital mechanics. Celestial Mech. 27, 65-77.

Bond, V.R., Allman, M.C., 1996. Modern Astrodynamics: Fundamentals and Perturbation Methods. Princeton University Press. Princeton, New Jersey.

Bowell, E., Virtanen, J., Muinonen, K., Boattini, A., 2002. Asteroid orbit computation. Asteroids III 1, 27-43.

Burdet, C.A., 1968. Theory of Kepler motion: the general perturbed two body problem. Zeitschrift für angewandte Mathematik und Physik ZAMP. 19, 345-368.

Campagnola, S., Skerritt, P., Russell, R.P., 2012. Flybys in the planar, circular, restricted, three-body problem. Celestial Mech. Dyn. Astron. $113,343-368$.

Chesley, S.R., Farnocchia, D., Brown, P., Chodas, P.W., 2014. Orbit estimation for late warning asteroid impacts: The case of 2014 AA, in: AAS/Division for Planetary Sciences Meeting Abstracts.

Deprit, A., 1976. Ideal frames for perturbed Keplerian motions. Celestial Mech. Dyn. Astron. 13, 253-263.

Deprit, A., Elipe, A., Ferrer, S., 1994. Linearization: Laplace vs. Stiefel. Celestial Mech. Dyn. Astron. 58, 151-201.

Flandro, G., 1966. Fast reconnaissance missions to the outer solar system utilizing energy derived from the gravitational field of jupiter1. Earth $108,6-1$.
Folkner, W.M., Williams, J.G., Boggs, D.H., Park, R.S., Kuchynka, P., 2014. The planetary and lunar ephemerides DE430 and DE431. Interplanetary Network Prog. Rep. 196, 1-81.

Folkner, W.M., 2014. Planetary ephemeris DE432. Technical Report. Jet Propulsion Laboratory. Pasadena, CA.

Gonzalo, J.L., Bombardelli, C., 2014. Asymptotic solution for the two body problem with radial perturbing acceleration. Adv. Astronaut. Sci. $152,359-377$.

Hansen, P., 1859. Auseinandersetzung einer zweckmssigen Methode zur Berechnung der absoluten Strungen der kleinen Planeten (1853). Printed in Abhandlungen der Kniglich Schsischen Gesellshaft der Wissenschaften, Bei S. Verlag. Leipzig, pp. 1-149.

Jenniskens, P., Shaddad, M., Numan, D., Elsir, S., et al., 2009. The impact and recovery of asteroid 2008 TC3. Nature 458, 485-488.

Jochim, E., 2012. The significance of the Hansen Ideal space frame. Astronomische Nachrichten 333, 774-783.

Kemble, S., 2006. Interplanetary Mission Analysis and Design. SpringerVerlag, Berlin Heidelberg New York

Kozai, Y., 1985. Secular perturbations of resonant asteroids. Celestial Mech. 36, 47-69.

Kustaanheimo, P., Stiefel, E., 1965. Perturbation theory of Kepler motion based on spinor regularization. J. Reine Angew. Math. 218, 204-219.

Laplace, P.S., 1799. Traité de mécanique céleste. Paris, Duprat et Bachelier. Reprinted in Euvres. Paris, Imprimerie royale, 1843.

Levi-Civita, T., 1920. Sur la régularisation du probleme des trois corps. Acta Math. 42, 99-144.

Milani, A., Chesley, S.R., Valsecchi, G.B., 2000. Asteroid close encounters with earth: risk assessment. Planet Space Sci. 48, 945-954.

Minovitch, M.A., 1961. A method for determining interplanetary free-fall reconnaissance trajectories. JPL Tec. Memo. 312 Pasadena, CA, pp. 1 130.

Palacios, M., Calvo, C., 1996. Ideal frames and regularization in numerical orbit computation. J. Astronaut. Sci. 44, 63-77.

Peláez, J., Hedo, J.M., de Andrés, P.R., 2007. A special perturbation method in orbital dynamics. Celestial Mech. Dyn. Astron. 97, 131-150.

Prado, A.F.B.A., 2007. A comparison of the patched-conics approach and the restricted problem for swing-bys. Adv. Space Res. 40, 113-117.

Rievers, B., Lämmerzahl, C., 2011. High precision thermal modeling of complex systems with application to the flyby and Pioneer anomaly. Annalen der Physik 523, 439-449.

Roa, J., Peláez, J., 2015. Frozen-anomaly transformation for the elliptic rendezvous problem. Celestial Mech. Dyn. Astron. 121, 61-81.

Roa, J., Peláez, J., 2015. Orbit propagation in Minkowskian geometry. Celestial Mech. Dyn. Astron., in press

Ross, S.D., Scheeres, D.J., 2007. Multiple gravity assists, capture, and escape in the restricted three-body problem. SIAM J. Appl. Dyn. Syst. $6,576-596$.

Sperling, H., 1961. Computation of Keplerian conic sections. ARS J. 31, 660-661.

Urrutxua, H., Pelaez, J., 2012. High fidelity propagation of asteroids using dromo-based techniques. In: 5th International Conference on Astrodynamics Tools and Techniques. ESA/ESTEC, The Netherlands.

Urrutxua, H., Sanjurjo-Rivo, M., Pelaez, J., 2013. Dromo propagator revisited. Adv. Astronaut. Sci. 148, 1809-1828.

Valsecchi, G., Milani, A., Gronchi, G., Chesley, S., 2003. Resonant returns to close approaches: analytical theory. Astron. Astrophys. 408, $1179-1196$. 